\title{
Théologiques
}

Théologiques

\section{La Cène}

\section{L'avant-dernière, la dernière, la première}

\section{Olivier Bauer}

Volume 23, numéro 1, 2015

\section{Derniers repas}

URI : https://id.erudit.org/iderudit/1040864ar

DOI : https://doi.org/10.7202/1040864ar

Aller au sommaire du numéro

\section{Éditeur(s)}

Faculté de théologie et de sciences des religions, Université de Montréal

ISSN

1188-7109 (imprimé)

1492-1413 (numérique)

Découvrir la revue

Citer cet article

Bauer, O. (2015). La Cène : l'avant-dernière, la dernière, la première.

Théologiques, 23(1), 15-37. https://doi.org/10.7202/1040864ar
Résumé de l'article

Il est d'usage de qualifier le repas que Jésus prend avec ses disciples la veille de son arrestation de " dernier repas ». Or, il n'en est rien. Une lecture attentive des évangiles de Luc et de Jean montre que Jésus mange encore après sa mort : au moins du poisson, peut-être du pain et du miel. Un tel constat pourrait relever de l'anecdote et le travail qui y conduit d'un pur souci d'érudition. Sauf qu'ils ont des conséquences pratiques et des impacts contemporains. Car l'insistance sur une « dernière cène » où Jésus partage le pain et le vin a conduit l'Église chrétienne à survaloriser l'eucharistie comme seul repas liturgique, alors que le christianisme primitif semble avoir fait de tous les repas l'occasion de rencontrer le Ressuscité.
Ce document est protégé par la loi sur le droit d'auteur. L'utilisation des services d'Érudit (y compris la reproduction) est assujettie à sa politique d'utilisation que vous pouvez consulter en ligne.

https://apropos.erudit.org/fr/usagers/politique-dutilisation/ 


\title{
La Cène
}

\section{L'avant-dernière, la dernière, la première}

\author{
Olivier Bauer" \\ Théologie pratique \\ Université de Lausanne
}

Même si ce n'est pas ainsi que le nomment les évangiles et la première épître de Paul aux Corinthiens, on ${ }^{1}$ considère le repas que Jésus prend peu avant son arrestation ( «la Cène ${ }^{2}$ », selon le terme consacré, celui que j'utiliserai dans cet article) comme son dernier repas. Or, sur ce point comme sur d'autres, les quatre évangiles ne sont pas unanimes. Dans ceux qui se revendiquent de Luc et de Jean, même mort, Jésus mange encore! Ou plutôt, ils racontent que Jésus mange encore après sa mort, justement pour affirmer qu'il n'est pas, ou qu'il n'est plus, tout à fait mort.

En soi, la thématisation des derniers repas de Jésus peut intéresser les spécialistes du Nouveau Testament. Mais plus largement, elle me paraît

* Olivier Bauer est professeur ordinaire à la Faculté de théologie et de sciences des religions à l'Université de Lausanne. Il est directeur de l'Institut lémanique de théologie pratique. Ses recherches portent notamment sur le rôle des six sens dans la transmission de la foi (ritualités, éducation de la foi), sur le sport et la religion (sport comme religion, aumônerie des milieux sportifs), ainsi que sur l'alimentation et la spiritualité (impact des religions sur les habitudes alimentaires, spiritualisation de l'alimentation). Il rédige actuellement un ouvrage d'érudition sur les aliments figurant sur les images médiévales de la Cène. Il a récemment publié (2017): Les cultes des protestant.e.s. Méthodes originales pour approcher les rites, Genève, Labor et Fides.

1. Ce «on» très général et très indéfini me semble adéquat pour désigner ainsi un inconscient collectif. En christianisme et dans les cultures influencées par le christianisme, la Cène est toujours «La Dernière Cène », "The Last Supper », "L'Ultima Cena", "Das letzte Mahl». On en trouve le signe le plus évident dans l'histoire de l'art (où «la Dernière Cène» est devenue un motif en soi). Mais c'est aussi une expression que même les biblistes utilisent volontiers. Il suffit de faire une recherche dans n'importe quel catalogue de bibliothèque pour en être convaincu.

2. Le terme "Cène» correspond au latin «cena» ( « dîner, repas principal vers 15 heures », Gaffiot 1934, 286). C'est le terme que la Vulgate utilise pour qualifier le repas que Jésus prend avant son arrestation (Jn 13,2 et 21,20; le verbe «cenare» en Lc 22,20) et celui que prennent les Corinthiens (nom en 1 Co 11,20.21; verbe en 1 Co 11,25). 
avoir un impact sur la question des repas liturgiques ou rituels en christianisme. Comment l'écrire? Il y aurait deux logiques: celle qui conduit d'une double survalorisation de la Cène (comme ultime repas de Jésus et comme institution d'un rite à célébrer) à une survalorisation de l'eucharistie (comme source et sommet de la vie chrétienne); et celle qui fait de tous les repas postpascaux (y compris les agapes) des «lieux de rencontre [...] possibles avec le Ressuscité " (Mainville 2007, 181), de toutes les nourritures (dont le pain, le fruit de la vigne, le poisson, peut-être le miel, mais pas seulement) des occasions de communion.

Je postule que, parce que la Cène n'est que l'avant-dernier repas de Jésus, l'eucharistie n'est que l'un des repas où il est possible de rencontrer le Ressuscité. Je vais soumettre ma thèse à deux épreuves, un peu formelles tant le résultat me paraît acquis. Je montrerai longuement que la Cène n'est pas forcément le dernier repas de Jésus et je rappellerai brièvement qu'il aura fallu attendre plus d'un siècle pour que le christianisme accorde un statut privilégié à l'eucharistie. L'originalité de ma réflexion et son intérêt réside, peut-être, dans la manière de lier le second constat au premier.

\section{Prendre part au dîner du Seigneur}

La plus ancienne lettre aux Corinthiens contient la première mention écrite d'un repas que Jésus aurait pris avant d'être arrêté. Mais ce récit vaut plus pour ce qu'il prescrit quant à la manière de partager un repas communautaire, que pour ce qu'il rappelle d'un événement de la vie de Jésus. Car de la Cène, Paul évoque à peine les circonstances, seulement un aliment et un ustensile. Il ne mentionne ni le menu du repas, ni le lieu où il se déroule, ni les convives qui le partagent. Il concentre son récit sur quelques gestes et sur quelques paroles qui les interprètent.

${ }^{23} \mathrm{Car}$ moi, j’ai reçu du Seigneur ce que je vous ai transmis : le Seigneur Jésus, dans la nuit où il allait être livré, prit du pain; ${ }^{24}$ après avoir rendu grâce, il le rompit et dit: "C'est mon corps, qui est pour vous; faites ceci en mémoire de moi. " ${ }^{25}$ Il fit de même avec la coupe, après le dîner, en disant: "Cette coupe est l'alliance nouvelle en mon sang; faites ceci en mémoire de moi, toutes les fois que vous en boirez.» $(1 \text { Co } 11,23-25)^{3}$

3. Tous les textes bibliques en grec sont cités d'après le Novum Testamentum Graece, $28^{\mathrm{e}}$ édition (Nestle et Aland 2012); les textes en français sont cités d'après la Nouvelle Bible Segond (Segond et Alliance biblique universelle 2002). Pour la traduction des termes grecs, nous avons utilisé trois dictionnaires: Bailly (1901), Bauer, Arndt et Gingrich (1957) et Carrez et Morel (1980). 
Au fond que transmet Paul ? Le fait que le Seigneur Jésus, à l'occasion

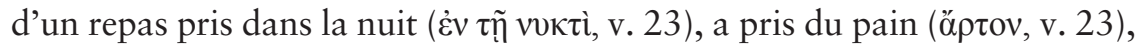

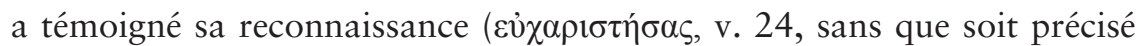
envers qui elle est manifestée), a rompu le pain, a donné le sens de ses

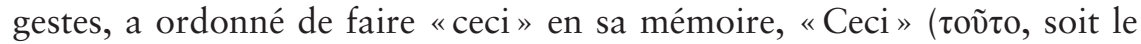
même terme utilisé dans l'expression «ceci est mon corps» v. 24) se rapportant au triple geste de prendre le pain, de rendre grâce et de rompre le pain et au fait de dire (y compris dans une belle mise en abyme de dire: «Faites ceci en mémoire de moi»), mais curieusement, pas de manger;

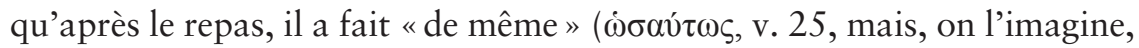
prendre la coupe et témoigner sa reconnaissance, pas la rompre) avec un

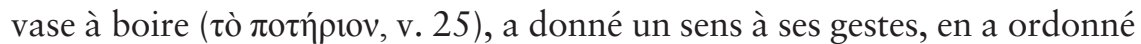
la répétition, avec une indication spécifique, celle de faire mémoire de lui toutes les fois que ses disciples en boiront. Rien de plus. Car ce qui intéresse Paul, lorsqu'il écrit aux Corinthiens, ce n'est pas le repas que Jésus aurait pris, mais le repas communautaire que prend «l'Église de Dieu qui est à Corinthe » (1 Co 1,2). Il en dénonce, de manière plutôt succincte, le déroulement ${ }^{4}$.

${ }^{17} \mathrm{Si}$ je ne vous félicite pas en formulant cette injonction, c'est que vous vous réunissez, non pas pour le meilleur, mais pour le pire. ${ }^{18} \mathrm{D}$ 'abord, j'apprends que lorsque vous vous réunissez en Église, il y a parmi vous des divisions - et je le crois en partie. ${ }^{19} \mathrm{Il}$ faut bien qu'il y ait aussi des dissensions entre vous, pour que ceux d'entre vous qui résistent à l'épreuve puissent se manifester. ${ }^{20}$ Donc, lorsque vous vous réunissez, ce n'est pas pour prendre part au dîner du Seigneur; ${ }^{21} \mathrm{car}$ au moment de manger, chacun se hâte de prendre son propre dîner, de sorte que l'un a faim tandis que l'autre est ivre. ${ }^{22} \mathrm{~N}$ 'avez-vous pas des maisons pour manger et boire? Ou bien méprisez-vous l'Église de Dieu en faisant honte à ceux qui n’ont rien? Que dois-je vous dire? Dois-je vous féliciter? Sur ce point, je ne vous félicite pas. (1 Co 11,17-22)

Ce court passage démontre l'existence d'une pratique de réunion communautaire dans le christianisme primitif, une réunion dans laquelle un repas tient une place importante. La communauté de Corinthe se réunit

4. «Les mauvaises manières de table des chrétiens corinthiens nous ont valu le passage le plus important du Nouveau Testament sur l'eucharistie et la version écrite la plus ancienne du récit de la Cène, datée d'entre le printemps 54 et le début de 57 . » (Genest 2005, 41). Sauf qu'il ne s'agit probablement pas de l'eucharistie, mais du «repas du Seigneur », ce qui n'est pas exactement pareil. 


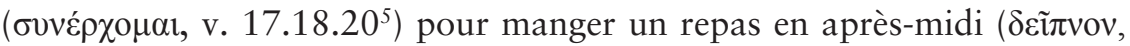

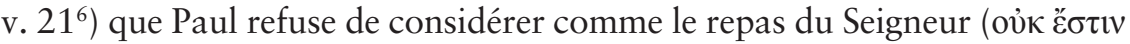
кטрıккòv $\delta \varepsilon \tilde{\pi} v$ ov, v. 20). Car chacun se presse de manger ce qu'il a apporté, de sorte que l'un a trop peu (il a faim: $\pi \varepsilon v v a \tilde{\alpha}, v .21$ ) et que l'autre a trop (il est ivre: $\mu \varepsilon \theta v ́ \varepsilon 1, v .21)$. Sans porter de jugement explicite sur cette pratique, Paul la renvoie au foyer: chez soi, voilà le lieu pour manger et pour boire

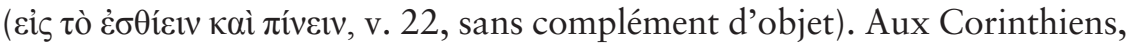
il rappelle que manger le repas du Seigneur n'est pas sans risques, que cela peut être, littéralement, une question de vie ou de mort.

${ }^{26}$ Car toutes les fois que vous mangez ce pain et que vous buvez cette coupe $^{7}$, c'est la mort du Seigneur que vous annoncez, jusqu'à ce qu'il vienne. ${ }^{27} \mathrm{C}^{\prime}$ est pourquoi celui qui mange le pain ou boit la coupe du Seigneur indignement sera coupable envers le corps et le sang du Seigneur. ${ }^{28}$ Que chacun s'examine plutôt lui-même, et qu'ainsi il mange du pain et boive de la coupe ${ }^{29}$ car celui qui mange et boit sans discerner le corps mange et boit un jugement contre lui-même ${ }^{8} .{ }^{30} \mathrm{C}^{\prime} e s t$ pour cela qu'il y a parmi vous beaucoup de malades et d'infirmes, et qu'un assez grand nombre se sont endormis dans la mort. (1 Co 11,26-30) ${ }^{9}$

Dans le chapitre 10 de sa première lettre aux Corinthiens, Paul avait déjà évoqué le pain et la coupe, mais sans les mettre en relation, ni avec la Cène, ni avec le repas du Seigneur.

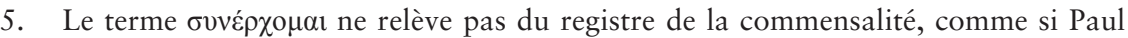
voulait signifier que ce n'est pas le «repas du Seigneur» que prennent les Corinthiens.

6. Carrez et Morel (1980): «repas principal pris le soir»; Bailly (1901): «repas de l'après-midi »; Bauer et al. (1957): «the main meal (toward) evening».

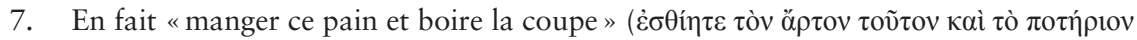
$\pi i ́ v \eta \tau \varepsilon$, v. 27). La précision «ce pain" pourrait indiquer l'utilisation d'un pain distinctif et spécifique. En revanche, l'utilisation du terme «coupe» laisse ouverte la question de la boisson qu'elle contient. Plus loin, Paul utilise une autre expression

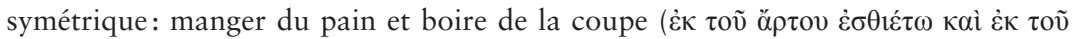

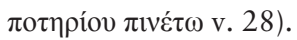

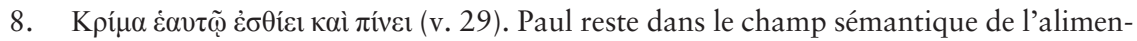
tation.

9. Paul distingue donc la communion chrétienne des repas rituels juifs et païens. "Ce qui diffère entre les trois repas rituels, c'est le vocabulaire sacrificiel. Il n'est employé qu'à propos d'Israël et des païens. [...] Ce qui est commun aux trois, c'est le vocabulaire de la communion, de la participation, de l'appartenance. [...] Ce qui est propre au repas rituel chrétien, outre le déplacement du convive de l'ordre de la divinité, c'est la relation instaurée entre les convives humains eux-mêmes, relation absente des deux autres repas.»(Genest 2005, 40-41). 
${ }^{14}$ Aussi, mes bien-aimés, fuyez l'idolâtrie. ${ }^{15} \mathrm{Je}$ vous parle comme à des gens avisés; jugez vous-mêmes de ce que je dis. ${ }^{16} \mathrm{La}$ coupe de bénédiction, sur laquelle nous prononçons la bénédiction, n'est-ce pas une communion au sang du Christ? Le pain que nous rompons, n'est-ce pas une communion au corps du Christ? ${ }^{17}$ Puisqu'il y a un seul pain, nous, la multitude, nous sommes un seul corps; car nous partageons tous le même pain. ${ }^{18}$ Voyez l'Israël selon la chair: ceux qui mangent des animaux offerts en sacrifice ne sont-ils pas en communion avec l'autel?

${ }^{19}$ Que dis-je alors? Que la viande sacrifiée aux idoles aurait de l'importance? Qu'une idole aurait de l'importance? ${ }^{20} \mathrm{Non}$, mais ce qu'on sacrifie, on le sacrifie à des démons et non à Dieu; or je ne veux pas que vous soyez en communion avec les démons. ${ }^{21}$ Vous ne pouvez pas boire à la coupe du Seigneur et à la coupe des démons; vous ne pouvez pas partager la table du Seigneur et la table des démons. ${ }^{22} \mathrm{Ou}$ bien voulons-nous provoquer la jalousie du Seigneur? Sommes-nous plus forts que lui ? (1 Co 10,14-22)

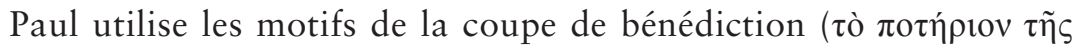

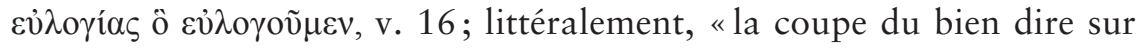
laquelle est dite du bien ») et du pain rompu, pour souligner la valeur théologique d'un tel repas. Paul mentionne aux Corinthiens deux rituels ali-

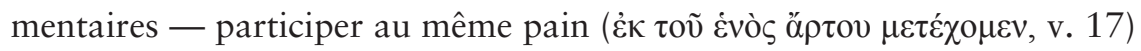

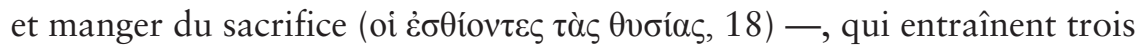
types de communion: une communion du sang et du corps du Christ

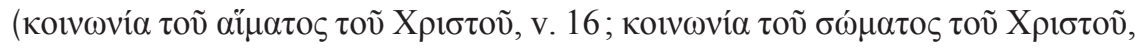

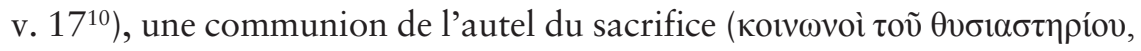

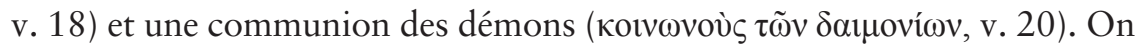

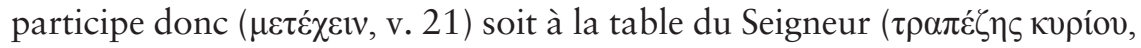

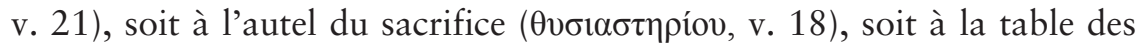

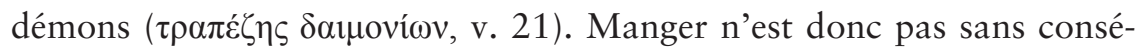
quence théologique. Si manger des animaux offerts en sacrifice, c'est être en communion avec l'autel, si sacrifier de la viande aux idoles (il n'est pas ici question de la manger), c'est être en communion avec les démons, alors, prononcer la bénédiction sur la coupe et rompre le pain, c'est être en communion au sang et au corps du Christ; partager le même pain, c'est, pour la multitude, être un seul corps. Mais cette communion ne peut se faire à l'occasion d'un sacrifice, forcément idolâtre, peu importe qu'il soit offert aux idoles du polythéisme ou sur l'autel des sacrifices juifs. La communion, qu'elle soit avec Dieu ou avec la multitude, ne passe plus par la

10. Dans cet ordre, le sang d'abord, le corps ensuite. 
viande, mais par du vin et du pain; elle n'exige plus de sacrifice mais une parole de bénédiction et un geste de partage ${ }^{11}$.

\section{Manger la Pâque}

En donnant un cadre narratif à la Cène, les quatre évangiles se montrent plus loquaces sur ce que fait Jésus avant son arrestation. Ils inscrivent le motif d'un repas marqué par un geste symbolique dans une séquence narrative qui met en scène les derniers jours de la vie de Jésus. Ces récits, jugés canoniques par les Églises chrétiennes ${ }^{12}$, constituent en quelque sorte les grands récits de la Cène. J'utilise à dessein le pluriel "grands récits» car le geste symbolique diffère selon les évangiles. Il en existe deux versions: les évangiles synoptiques (Matthieu, Marc et Luc ${ }^{13}$ ) y situent un partage de pain et de fruit de la vigne, l'évangile attribué à Jean y place un lavement des pieds. Mais quel que soit le geste, il a lieu lors d'un repas, un repas que Marc raconte ainsi :

${ }^{17}$ Le soir venu, il arrive avec les Douze. ${ }^{8}$ Pendant qu'ils étaient à table et mangeaient, Jésus dit: «En vérité, je vous le déclare, l'un de vous va me livrer, un qui mange avec moi. » ${ }^{19}$ Pris de tristesse, ils se mirent à lui dire l'un après l'autre: «Serait-ce moi ?" ${ }^{20} I l$ leur dit: "C'est l'un des Douze, qui plonge la main avec moi dans le plat. ${ }^{21}$ Car le Fils de l'homme s'en va selon ce qui est écrit de lui, mais malheureux l'homme par qui le Fils de l'homme est livré! Il vaudrait mieux pour lui qu'il ne soit pas né, cet homme-là!» ${ }^{22}$ Pendant le repas, il prit du pain et, après avoir prononcé la bénédiction, il le rompit, le leur donna et dit: «Prenez, ceci est mon corps.» ${ }^{23}$ Puis il prit

11. Selon 1 Co 8,1-12, consommer une viande de sacrifice ne présente aucun danger théologique: la nourriture n'a aucune valeur théologique et les dieux des païens sont de faux dieux. Mais cette liberté de consommer les viandes du sacrifice présente des risques éthiques. Les chrétiens issus du paganisme pourraient continuer à la manger non pas par goût, mais comme un tribut offert aux idoles. Devant ce risque, Paul prend une position radicale et renonce à consommer au moins la viande de sacrifice, et peut-être même simplement de la viande, comme le texte le laisse penser.

12. D’autres récits de ce repas n'ont pas été jugés canoniques et ne figurent donc pas dans le Nouveau Testament. Le plus étonnant se trouve dans le "Livre du coq", un récit éthiopien tardif. Jésus y ressuscite le coq rôti que l'hôtesse avait placé devant lui (Livre du Coq 4, 1-5, cité par Geoltrain, Kaestli, Roessli et Voicu 2005, 168-169).

13. Comme me le rappelle Alain Gignac (que je remercie pour la relecture attentive de cet article, en particulier pour sa révision très précise des termes grecs), on distingue deux traditions dans les Évangiles synoptiques: Luc d'un côté, que l'on rapproche de Paul notamment parce que les deux récits contiennent l'ordre de «faire ceci en mémoire de moi »; Matthieu et Marc de l'autre. 
une coupe et, après avoir rendu grâce, il la leur donna et ils en burent tous.

${ }^{24}$ Et il leur dit: "Ceci est mon sang, le sang de l'Alliance, versé pour la multitude. ${ }^{25}$ En vérité, je vous le déclare, jamais plus je ne boirai du fruit de la vigne jusqu'au jour où je le boirai, nouveau, dans le Royaume de Dieu.» ${ }^{26}$ Après avoir chanté les psaumes, ils sortirent pour aller au mont des Oliviers. (Mc 14,18-26 // Mt 26,21-30 // Lc 22,14-23; cf. Jn 13)

Les quatre évangiles donnent à la Cène un cadre dont Paul ne dit rien. Ils situent le repas à l'occasion de la Pâque juive, en font un repas d'adieu ${ }^{14}$ et précisent l'identité des convives: outre Jésus, ce sont les disciples (Mt 24,7, Mc 14,12 et Jn 13,5), «les Douze» (Mc 14,17) ou «les apôtres» (Lc 22,14), sans que ces différences ne paraissent significatives. Les évangiles synoptiques localisent le repas à Jérusalem (littéralement «dans la

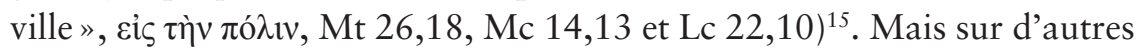
aspects de ce repas, les évangiles ne sont pas unanimes. Les récits présentent des différences dans leur contenu, certaines légères, d'autres substantielles ${ }^{16}$. À commencer, évidemment, par celui de Jean dont il serait trop long de montrer toutes les particularités. Mais même les différences entre les évangiles de Matthieu, de Marc et de Luc peuvent être notables. Chacun

14. Avec des différences selon les évangiles ( «le premier jour des Pains sans levain », Mt 24,17; "le jour des Pains sans levain", Mc 14,12 et Lc 22,7; "avant la fête de la Pâque ", Jn 13,1), des différences qui soulèvent un problème de datation difficile à résoudre pour qui voudrait traiter ce récit comme un événement historique. Dans le calendrier juif, le «jour où l'on immole la Pâque » n'est pas «le premier jour des pains sans levain ". C'est le 14 du mois de Nisan que les Juifs sacrifient l'agneau de la Pâque et qu'ils le mangent au cours du repas du Seder. Et c'est le lendemain, le 15 Nisan, que commence la fête des pains sans levain ou des Azymes. Humphreys (2011) tente d'harmoniser les dates, en faisant débuter le jour juif le matin et non le soir.

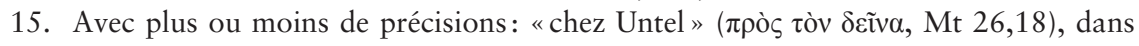

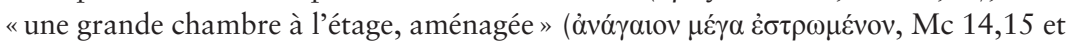

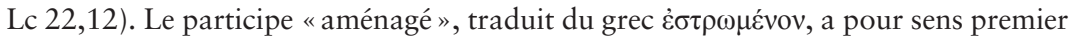
"étendu », notamment quand il s'agit d'un lit, d'un tapis ou d'une couverture. Ce qui donne une indication sur la manière dont le repas a été pris.

16. On constate aussi des différences entre Paul et les évangiles synoptiques: "For example, the bread-saying in Paul has an interpretation attached to the metaphor of "body"; the saying in Mark does not. The cup-saying in Paul equates the sharing of cup with covenant which is ratified by blood. The saying in Mark equates the content, the wine, with blood which is interpreted both in terms of covenant and martyrdom. Only in Paul is there a command to repeat the ceremony, and it is connected with the theme of memorial, in Mark, on the other hand, the text is enclosed in a narrative context, just as are the other Jesus stories, without any obvious sense that it is intended to mirror liturgical practice in the Markan community, or, if it is, how it is to do so.” (Smith et Taussig 1990, 40). 
insère dans sa narration des épisodes qui lui sont propres ( $L u c$ inclut dans le dernier repas, une controverse sur la hiérarchie parmi les apôtres, Lc 22,24-30) ou qu'ils partagent (Matthieu et Marc racontent un autre repas au cours duquel une femme parfume les pieds de Jésus, Mt 26,6-13 et Mc 14,3-9). Et quand ils s'accordent sur un événement, ils peuvent l'exprimer de manière différente: en Matthieu et en Marc, Jésus dit du bien du pain

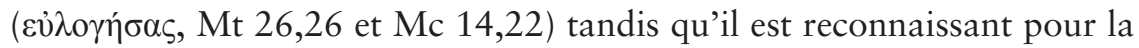

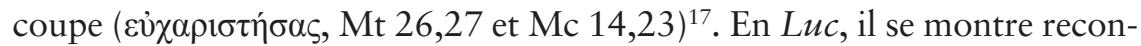

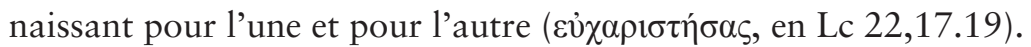

Faisant abstraction des paroles et des gestes (ce qui n'a évidemment aucun sens, puisque c'est dans ces paroles et ces gestes que se trouve la raison d'être des récits de la Cène), on peut dire que les quatre récits

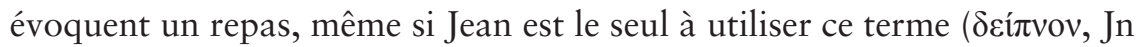
13,2.4) et que les évangiles synoptiques recourent à différentes périphrases pour le désigner: manger la Pâque (

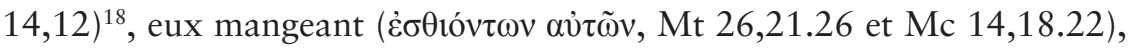

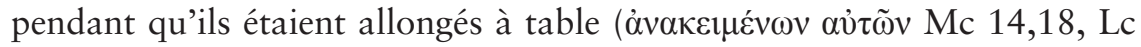

17. Paul utilise la formulation inverse: Jésus dit du bien de la coupe $(1$ Co 10,16$)$ et se montre reconnaissant pour le pain (1 Co 11,24).

18. Les évangiles font de Jésus un Juif qui mange la Pâque: "J'ai vivement désiré manger cette Pâque avec vous, avant de souffrir » $($ Lc 22,15). Ce qui n'est pas anodin: "The Passover is a metonymic food. If access to the Passover signifies inclusion in the collective body of 'Israel, ' then eating the metonymic food with peers can be understood as a communal ritual of identity enactment and maintenance." (Rosenblum 2010, 163). Mais Luc, aussitôt, le désolidarise de la communauté juive: «car, je vous le dis, je ne la mangerai plus jusqu'à ce qu'elle soit accomplie dans le royaume de Dieu.» Lc 22,16. Ce qui n'est pas plus anodin: “Concomitantly, refusal to ingest the Passover can be construed as an actor social separation and group disintegration, alone might read Jesus' abstention from the Passover in Luke 22: 15-16. » (Rosenblum 2010, 163). Au plan littéraire ou narratif, le caractère pascal du repas est sujet à caution: "Un programme narratif très clair, exprimé dès les premiers versets: manger la Pâque. Mais, une fois le groupe à table, il ne restera des préparatifs de la Pâque juive et des éléments caractéristiques que la bénédiction et l'action de grâces sur le pain et la coupe et le chant des psaumes à la sortie de la salle, soit, paradoxalement, des éléments communs à tous les repas. À partir des gestes et paroles de Jésus sur le pain, le repas pascal (et ses figures propres) est recatégorisé en un repas d'alliance. Ce dernier constitue également un repas d'adieu définitif, profilé sur l'évocation du repas eschatologique dans le royaume de Dieu. (Genest 2005, 36-37). Sans oublier que les récits de la Cène reprennent plutôt une structure grecque: "There were two major courses in a banquet, the dipnoan ('supper' or 'banquet'), which was the meal proper, followed by the symposium ('symposion'), which was the drinking party. This form is reflected in the Lord's Supper traditions in the New Testament in which the wine is drunk 'after supper'.” (Smith et Taussig 1990, 25). 


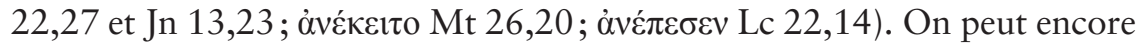
dire qu'ils mentionnent deux éléments de la vaisselle utilisée: une coupe (Mt 26,27, Mc 14,23 et Lc 22,17.20) et un plat ou une assiette ( $\tau \rho v \beta \lambda i ́ o v$ en Mt 26,23 et Mc 14,20) au contenu indéterminé. On peut enfin dire qu'ils témoignent de peu d'intérêt pour le menu du repas. Dans les évangiles, on mange, mais la plupart du temps sans que soit précisé quels aliments ( $\varphi \gamma \varepsilon \varepsilon \tilde{v}$ est utilisé sans complément d'objet direct en Mt 26,21.26, Mc 14,18(2x).22 et Lc 22,8) ou alors en indiquant que c'est la Pâque que mangent les convives, mais sans autre précision sur le menu (Mc 14,12 et Lc $22,11.15)^{19}$. En fait, seuls Matthieu et Jean indiquent des aliments. Chez

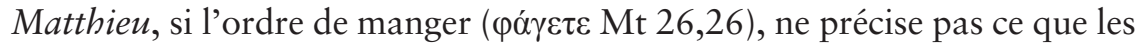
disciples doivent manger, le contexte permet de comprendre qu'ils doivent manger le pain (mentionné en Mt 26,26, Mc 14,22 et Lc 22,1920) qu'il vient de rompre et de leur donner. Et Jean indique explicitement un aliment, celui que Judas avale: une bouchée de viande ou de pain ( $\varphi \omega \mu$ เó, Jn $13,26.27 .30)$ que Jésus plonge ( $\beta \alpha \psi \omega$, Jn 13,26.27, sans qu'il soit précisé dans quoi) et lui tend ${ }^{21}$. On boit aussi, à partir de la coupe ( $\dot{\xi} \xi \alpha$ đovov, $\mathrm{Mt}$ 26,27 et Mc 14,23), sans autre précision, quand bien même le contexte

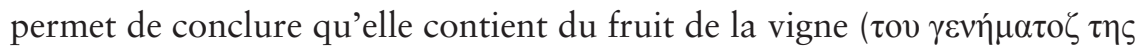

19. Il est très aléatoire de prétendre vouloir reconstituer le menu du repas pascal à l'époque de Jésus. "While it has often been assumed that a reconstructed picture of Jewish meals based on the rabbinic evidence can be used as a starting-point from which formal and other characteristics of early eucharistic meals can be derived, it now seems that we cannot really systematize the evidence of the Mishna and other rabbinic texts in such a way as to project a supposedly fixed liturgy of (e.g.) the Seder back into the first century with an expectation of setting the two pictures together. Even those who still value the mutual light the two may shed are increasingly cautious about assuming a fixed pattern of either Seder or eucharistic meal at such an early point." (McGowan 1999, 53).

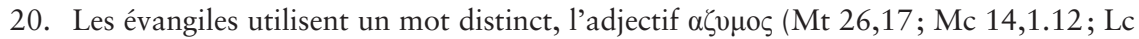
$22,1.7$, littéralement «sans levain»), pour évoquer les «pains sans levain» dont la fête fournit l'occasion du repas. L'utilisation de deux mots distincts pourrait témoigner en faveur de l'usage d'un pain levé lors du repas de Jésus. L'évangile attribué à

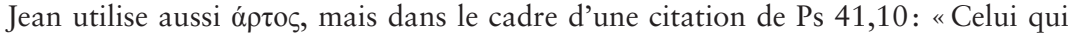
mange mon pain a levé son talon contre moi. » (Jn 13,18.) Notons que dans le Tanakh, le terme utilisé est לְֶ (dont le sens premier est "pain" mais qui signifie

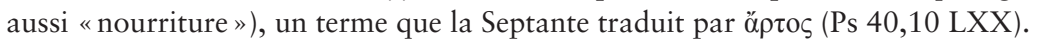

21. Notons que c'est aussi par la bouche que le satan (ó $\sigma \alpha \tau \alpha v \tilde{\alpha} \varsigma$, Jn 13,27 avec un article défini et une minuscule) entre en Judas et à la suite de la bouchée. Faut-il alors en faire un aliment? 
$\alpha \mu \pi \varepsilon ́ \lambda$ ov, mentionné en Mt 26,29, Mc 14,25 et Lc 22,18²2). Et Jésus fait partie des mangeurs et des buveurs. Implicitement, Jésus est inclus dans les

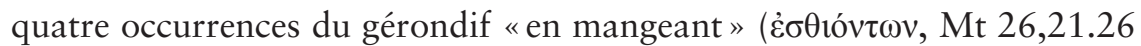
et Mc 14,18.22). Explicitement, il est sujet ou fait partie des sujets du

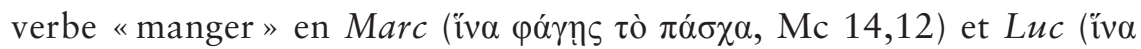

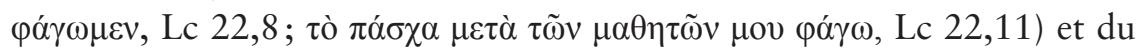

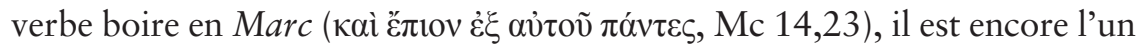

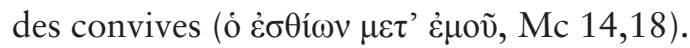

Si la Cène représente donc bien un repas de Jésus, s'agit-il pour autant de son dernier repas? Les évangiles synoptiques terminent leurs récits de la Cène par une promesse de Jésus qui s'engage à ne plus boire du fruit de la vigne (Mt 26,29 // Mc 14,25 et Lc 22,18 ${ }^{23}$ ) et en Luc, en plus, à ne plus manger la Pâque (Lc 22,16). L'annonce d'un jeûne de solide et de liquide accréditerait la thèse que la Cène soit son dernier repas. Pourtant, les trois évangiles poursuivent la phrase de Jésus, et apportent la nuance que sa promesse n'est que temporaire: un jour, dans le royaume de son père, Jésus recommencera à boire du fruit de la vigne; il le boira nouveau, avec ses

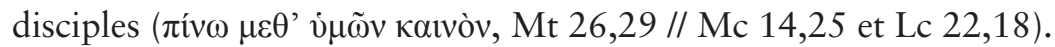

Mais, je le reconnais, cela ne suffit pas à faire un repas, tout au plus un apéritif, qui de plus reste encore à venir. Ce qui fait que la Cène reste encore le dernier repas de Jésus.

\section{Prendre du vin aigre}

Dans les récits de la crucifixion, deux épisodes évoquent quelque chose à boire, peut-être un aliment, peut-être un médicament. Matthieu et Marc indiquent que les soldats qui s'apprêtent à crucifier Jésus lui proposent du vin mêlé de fiel (oĩvov $\mu \varepsilon \tau \grave{\alpha} \chi 0 \lambda \tilde{n} s$, Mt 27,3424) ou du vin aromatisé de

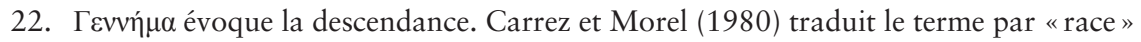
ou «fruit », Bailly (1901) indique comme sens possible «enfant », « rejeton », "produit de la nature ou de l'éducation» et Bauer et al. (1957) propose «that which is produced or born (of living creatures) ». L'utilisation d'un terme associé à la filiation laisse penser que ce fruit de la vigne, qui se trouve dans un «vase à boire» et que Jésus ordonne de boire ( $\pi \dot{\varepsilon} \varepsilon \tau \varepsilon \varepsilon \dot{\varepsilon} \xi \alpha$ a $\tau o \tilde{v}$ en Mt 26,27 et Mc 14,23) serait plutôt du jus de raisin non fermenté que du vin, qui réclame un travail humain.

23. Mais les deux autolimitations ne portent pas sur le fait de manger en général, mais de manger la Pâque (avec toute la polémique antijuive que cela sous-entend) ni sur le fait de boire en général, mais de boire du, ou même de ce, fruit de la vigne.

24. Pour oĩvos, Bailly (1901) indique le vin, mais aussi toute boisson fermentée, dont le vin de palmier; Bauer et al. (1957) précise qu'il s'agit du vin, «normally the fermented juice 


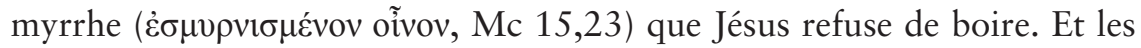
quatre évangiles mentionnent que quelqu'un (seul $L u c$ précise qu'il s'agit

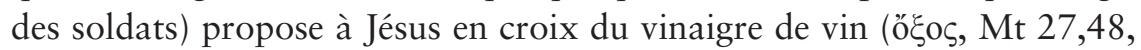
Mc 15,36, Lc 23,37 et Jn 19,29[2x].30). Dans les évangiles synoptiques, Jésus ne le boit pas, puisqu'il meurt aussitôt. Mais il en va autrement en Jean.

${ }^{28}$ Après cela, pour que l'Écriture soit accomplie, Jésus, sachant que déjà tout était achevé, dit: J'ai soif. ${ }^{29} \mathrm{Il}$ y avait là un récipient plein de vin aigre. On fixa à une tige d'hysope une éponge imbibée de vin aigre et on l'approcha de sa bouche. ${ }^{30}$ Quand il eut pris le vin aigre, Jésus dit: Tout est achevé. Puis il baissa la tête et rendit l'esprit. (Jn 19,28-3025

Mais j'en conviens, boire un peu de vinaigre ne représente toujours pas un nouveau repas. Ce qui fait que la Cène reste, à ce stade narratif, encore le dernier repas de Jésus.

\section{Déjeuner}

On pourrait penser que la mort de Jésus règle définitivement la question du dernier repas de Jésus. Car après tout, s'il n'a pas pris de repas entre son arrestation et son exécution, le repas qu'il a pris avant son arrestation devient, ipso facto, son dernier repas. Sauf que dans les évangiles, Jésus n'est pas un être humain comme les autres et que, pour un certain nombre de témoins, sa mort ne met pas fin à son existence. Ainsi deux évangiles, ceux de Luc et de Jean, incluent de la nourriture dans leurs christophanies, c'est-à-dire dans les récits qu'ils font des apparitions de Jésus ressuscité. Luc et Jean mettent chacun en scène un récit où Jésus est celui qui donne à manger, $L u c$ ajoute un second épisode où Jésus est celui qui demande à manger.

of the grape ». Rappelons que ce terme oĩvo n'apparaît ni dans la première lettre aux

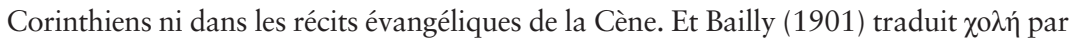
«bile", "fiel » mais aussi "venin d'un serpent»; Bauer et al. (1957) en élargit le sens à « of a substance w. an unpleasant taste ».

25. Ce bref récit a pour fonction première de rendre l'exécution de Jésus conforme à ce qu'évoque le Ps 69,22: «Ils mettent du poison dans ma nourriture, et, pour apaiser ma soif, ils me font boire du vinaigre ». "Poison» traduit ce que le Tanakh exprime

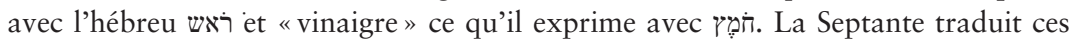

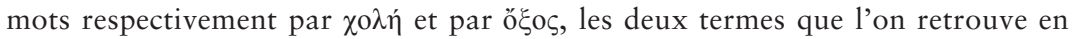
Matthieu. 


\section{1 «Puis il le rompit et le leur donna»}

Luc raconte comment deux disciples marchent vers Emmaüs sans reconnaître Jésus ressuscité qui s'est joint à eux. Le malentendu dure tout au long du chemin et ne se dissipe qu'au moment où les deux disciples proposent à Jésus de s'attabler avec eux.

${ }^{28}$ Lorsqu'ils approchèrent du village où ils allaient, il parut vouloir aller plus loin. ${ }^{29}$ Mais ils le pressèrent, en disant: Reste avec nous, car le soir approche, le jour est déjà sur son déclin. Il entra, pour demeurer avec eux. ${ }^{30}$ Une fois installé à table avec eux, il prit le pain et prononça la bénédiction; puis il le rompit et le leur donna. ${ }^{31}$ Alors leurs yeux s'ouvrirent et ils le reconnurent; mais il disparut de devant eux. ${ }^{32} \mathrm{Et}$ ils se dirent l'un à l'autre: Notre cœur ne brûlait-il pas en nous, lorsqu'il nous parlait en chemin et nous ouvrait le sens des Écritures? ${ }^{33}$ Ills se levèrent à ce moment même, retournèrent à Jérusalem. (Lc 24,28-33)

Cette rencontre pourrait être l'occasion d'un repas. Les trois convives

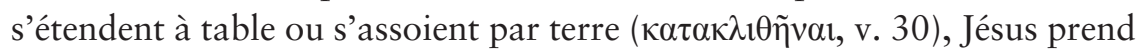

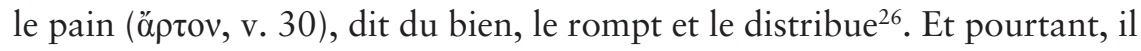
n’y a pas de repas! Personne ne mange, ni Jésus qui disparaît, ni les deux disciples qui se lèvent pour retourner à Jérusalem. Car la fraction du pain a suffi. Elle a rempli sa fonction. Elle a permis aux deux disciples de recon-

26. Mainville $(2007,192)$ est d'avis que «Luc recrée l'expérience de la Cène en reprenant

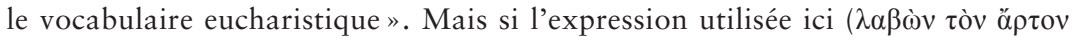

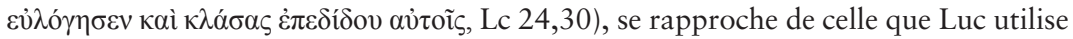

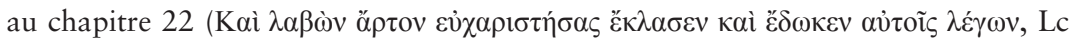
22,19), elle en diffère sur des détails (le choix et le temps de certains verbes) mais aussi sur un point essentiel: le chapitre 24 ne fait aucune mention de la coupe, pourtant partie intégrante de la Cène, et plutôt deux fois qu'une chez Luc. Mainville affirme aussi que cette christophanie «indique au croyant les lieux de rencontre désormais possibles avec le Ressuscité: les Écritures et le Mémorial de la Cène.» (2007, 181). Mais, dans l'œuvre de Luc, les expressions «rompre le pain» ou «fraction du pain» désignent de manière générale les repas partagés (Ac 2,42; 20,7.11). Et Ac 27 décrit ainsi l'action de Paul: «Il a pris du pain, il a rendu grâce à Dieu

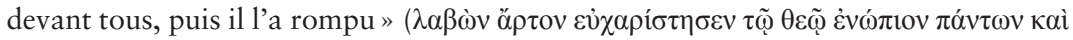
$\kappa \lambda \alpha ́ \sigma \alpha \varsigma$, v. 35), une formule qui introduit un vrai repas, comme l'indique l'exhorta-

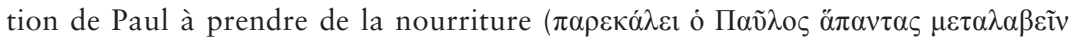

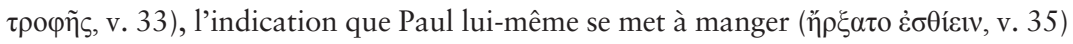

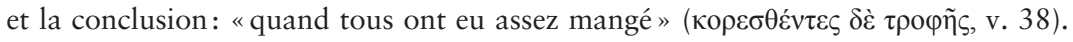
Odette Mainville signale en note l'interprétation large proposée par Roger David Aus: Luc 24 "invite à la commensalité et marque la nécessité d'y intégrer les étrangers» $(2007,181)$. La référence vient de Aus (2000, 137-234 et 215-217). 
naître la présence de Jésus ressuscité. Ils pourront raconter aux Onze «comment il s'était fait reconnaître d'eux en rompant le pain» (v. 35 ${ }^{27}$.

Au fil de notre enquête narrative, Jésus n'a donc toujours pas pris de nouveau repas. Et la Cène reste toujours son dernier repas.

\section{2 "Jésus vient, prend le pain et le leur donne, ainsi que le poisson»}

Parmi ses christophanies, Jean propose "une excursion de pêche" (Mainville 2007, 36), construite sur un scénario très proche du récit de Luc. Après une nuit passée sur la mer de Tibériade sans avoir rien attrapé, sept disciples voient un homme qui se tient debout sur le rivage. Ils ne reconnaissent pas Jésus. Il faut que celui-ci leur donne un signe, une pêche exceptionnelle, pour qu'ils l'identifient et se décident à le rejoindre.

${ }^{9}$ Lorsqu'ils furent descendus à terre, ils voient là un feu de braises, du poisson posé dessus, et du pain. ${ }^{10}$ Jésus leur dit: Apportez quelques-uns des poissons que vous venez de prendre. ${ }^{11}$ Simon Pierre monta dans le bateau et tira à terre le filet, plein de cent cinquante-trois gros poissons; et quoiqu'il y en eût tant, le filet ne se déchira pas. ${ }^{12}$ Jésus leur dit: Venez déjeuner. Aucun des disciples n'osait lui demander: Qui es-tu, toi ? Car ils savaient que c'était le Seigneur. ${ }^{13}$ Jésus vient, prend le pain et le leur donne, ainsi que le poisson. (Jn 21)

À ce point, le récit s'interrompt et Jean ajoute une incise: ${ }^{14}{ }^{14}$ 'était déjà la troisième fois que Jésus se manifestait à ses disciples depuis qu'il s'était réveillé d'entre les morts. Et le récit se poursuit, bref, laconique: " ${ }^{15}$ Après qu'ils eurent déjeuné, Jésus dit à Simon Pierre [...]».

Cette fois, il y a bien repas. Ils mangent, ils prennent le repas du milieu

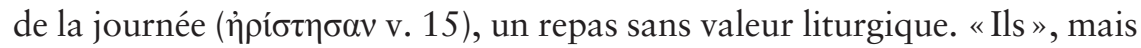
qui ? Les disciples sans aucun doute, mais Jésus aussi, qui ne peut pas être exclu des sujets du verbe "déjeuner ». Alors si Jésus mange, la Cène n'est plus que son avant-dernier repas.

Et les aliments ne sont pas insignifiants. Jusqu'ici Jésus avait mangé du pain et bu du fruit de la vigne et du vinaigre. En Jean, le binôme pain et poisson renvoie non pas à la Cène (Jean n'y mentionne aucun aliment, sinon une bouchée), mais au récit d'un autre repas, celui où Jésus nourrit une foule «d'environ cinq mille hommes", en multipliant les «cinq pains

27. En 1 Co 11,25, l'ordre de Jésus de faire «ceci en mémoire de [lui]» porte sur trois gestes, prendre le pain, dire du bien, rompre le pain, peut-être sur le fait de dire, mais pas sur le fait de manger le pain. 


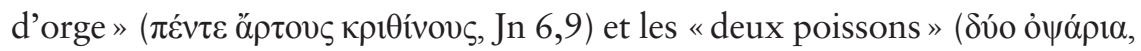
Jn 6,9) d'un jeune garçon. Sur les rives de la mer de Tibériade, ce sont à nouveau les mêmes aliments que Jésus donne ${ }^{28}$.

\section{3 «Il les mangea devant eux»}

Du poisson, il en est aussi question dans le denier texte que je vais examiner, le récit de la dernière apparition de Jésus ressuscité, en $L u c$.

${ }^{36}$ Comme ils disaient cela, lui-même se présenta au milieu d'eux et leur dit: Que la paix soit avec vous! ${ }^{37}$ Saisis de frayeur et de crainte, ils pensaient voir un esprit. ${ }^{38}$ Mais il leur dit: Pourquoi êtes-vous troublés? Pourquoi des doutes vous viennent-ils ? ${ }^{39}$ Regardez mes mains et mes pieds, c'est bien moi; palpez-moi et regardez; un esprit n'a ni chair ni os, comme vous voyez que j'en ai. ${ }^{40} \mathrm{Et}$ en disant cela, il leur montra ses mains et ses pieds. ${ }^{41} \mathrm{Comme}$, dans leur joie, ils ne croyaient pas encore et qu'ils s'étonnaient, il leur dit: Avez-vous ici quelque chose à manger ? ${ }^{42}$ Ils lui présentèrent un morceau de poisson grillé [et un rayon de miel] ${ }^{29} .{ }^{43}$ Il [les] prit et [les] mangea devant eux. (Lc 24, 36-43)

Pour prouver qu'il n'est pas un fantôme, Jésus sollicite la vue de ses disciples (il montre ses blessures), le toucher (il leur enjoint de palper sa chair et ses os) et, preuve ultime, il démontre qu'il est capable de manger ce que lui offrent les disciples. Et que mange-t-il ? Un morceau de poisson

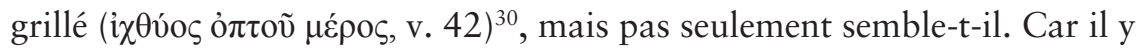

28. Mais quel poisson donne-t-il ? La question n'est pas aussi anodine qu'elle peut paraître. Odette Mainville $(2007,216)$ affirme que «le Ressuscité alimente [les disciples] d'une nourriture qu'ils n'ont pas eux-mêmes produite». Mais le texte grec

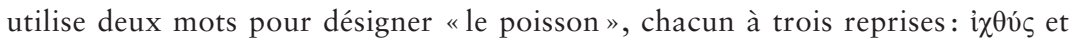
óuópıov. Jean utilise i $\chi \theta 0$ ć aux versets 6,8 et 11, exclusivement pour désigner les poissons pêchés par les disciples. Il utilise ỏyópıov (Bailly (1901): "petits mets ", particulièrement «petits plats de poisson"; Bauer et al. (1957): "'cooked food' eaten w. bread» pour désigner le poisson qui grille sur les braises (v. 9), celui que Jésus demande aux disciples d'apporter (v. 10) et celui que Jésus leur donne (v. 13), ce qui laisse penser que Jésus donne aux disciples tout à la fois un poisson qu'ils ont et n'ont pas pêché, une nourriture qu'ils ont et n'ont pas produite.

29. "Adjonction tardive» (Vouga 2011, 257), «la leçon "rayon de miel" [...] est attestée par d'excellents témoins.» (Vogel 1980, 89, note 10)

30. Cette prolifération de poisson pourrait correspondre au fait que le poisson ait figuré au menu de certains repas rituels du christianisme primitif. Mais il convient de rester prudent. "The prominence of fish in the Gospels feeding stories and the use of fish in early Christian art and inscriptions raise the question of the possible use of fish in ritual meals. » (McGowan 1999, 127). Mais le poisson vaut aussi pour sa valeur 
a peut-être plus à manger. Une variante du texte ajoute, après le poisson

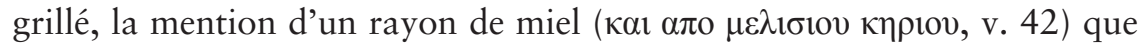
Jésus prend et qu'il mange aussi. De ce binôme poisson et miel, François Vouga tire deux conséquences possibles, sans trancher entre les deux.

- La mention du miel pourrait renforcer l'aspect sacramentel du récit. Car si le poisson évoque l'eucharistie ${ }^{31}$, le miel renvoie au baptême ${ }^{32}$. - Mais, de manière tout aussi intéressante, la mention de poisson et de miel pourrait simplement évoquer un plat apprécié dans le monde romain. Luc signalerait ainsi la joie des retrouvailles entre Jésus et ses disciples, et inviterait peut-être même à faire de même, en mémoire de ce repas ${ }^{33}$.

symbolique, qui précède et excède le christianisme. «Le poisson a constitué, bien avant le christianisme, un aliment sacré. Dans le monde antique, il symbolise la puissance virile (ichtyphalles); dans le judaïsme, il inaugure les temps messianiques; les chrétiens l'ont repris dans sa signification eschatologique. » (Vogel 1980, 87)

31. Vouga tente de reconstruire le lien peu évident qui lie le poisson à l'eucharistie. «Le poisson rappelle le récit de la multiplication des pains (Lc 9, 10-17), qui préfigure lui-même la dernière Pâque de Jésus et l'institution du "repas du Seigneur ", que les Actes désigneront par la métonymie du pain rompu (Lc 22, 14-20). » (Vouga 2011, 257). Maurice Vloberg apporte plus de précisions: «Le symbole tourna tout de suite au sens eucharistique. Le Poisson divin vivant dans nos eaux mortelles et pleines d'amertume, désigna le miracle des miracles, la transsubstantiation des espèces du sacrifice.» (Vloberg 1946, 12-13). Il ajoute que «le symbole du Poisson, d'une idéographie si claire à l'origine, se complique plus tard sous l'influence de textes scripturaires. Du "Poisson vivant", expression de la divinité toujours présente et puissante du Sauveur, une transition subtile mène à l'image du "Poisson cuit", expression des humiliations de l'Homme-Dieu. Le rapport entre le Christ souffrant et le poisson mis sur le gril fut résumé en cette formule à succès: "Piscis assus, Christus passus", dit saint Augustin, qui avait un faible pour l'allitération, sinon pour le jeu de mots.» (Vloberg 1946, 13-14). D'ailleurs, ce sont des poissons qui figurent sur la plus ancienne image de la Cène, la mosaïque byzantine de la basilique San Appolinare Nuovo à Ravenne. Y figurent, posés sur un plat, deux gros poissons entiers, avec leur tête et leur queue.

32. «Le miel prend place, dès la fin du $\mathrm{II}^{\mathrm{e}}$ ou au début du $\mathrm{III}^{\mathrm{e}}$ siècle, dans certaines liturgies de baptême. Tertullien témoigne d'une pratique selon laquelle on présentait du miel au baptisé qui venait de ressusciter à une vie nouvelle.» (Vouga 2011, 257). Le miel était probablement parfois ajouté au vin de l'eucharistie. C'est au moins ce que laisse penser le fait que le synode diocésain d'Auxerre à la fin du VIe siècle, rappelle «qu'il est interdit d'utiliser du vin miellé.» (Caseau 2002, 125)

33. «Peut-être faut-il cependant inverser la relation de cause à effet. Le poisson au miel est une préparation appréciée de l'Antiquité, et la fête gastronomique à laquelle s'invite le Ressuscité correspond, dans l'évangile de Luc, au veau gras que le père met à rôtir pour le retour de son fils prodigue (Lc 15,23). Les disciples célèbrent le retour de Jésus - dans la joie étonnée de le retrouver — et, à la différence des récits sem- 
Il n’y a plus aucun doute. Après qu'il a été arrêté, crucifié et ressuscité, Jésus mange encore. La Cène n'est plus que son avant-dernier repas.

\section{Rompre le pain}

La démonstration me semble convaincante. Chez Luc et chez Jean, la Cène n'est pas le dernier repas de Jésus, mais l'avant-dernier; elle sera même son antépénultième repas, lorsque viendra le règne de Dieu. Et que «même mort, Jésus mange encore» me paraît doublement significatif. En plus d'indiquer la résurrection (en ce sens, Jésus n'est plus vraiment mort, ce qui justement lui permet de manger encore), ces récits témoignent d'une vision plutôt large des repas et des aliments pouvant servir de «lieux de rencontre désormais possibles avec le Ressuscité » (Mainville 2007, 181). On peut le rencontrer chaque fois que l'on mange et dans la consommation des nourritures ordinaires: le pain et le fruit de la vigne évidemment, le poisson et le miel également, mais aussi les laitages, lait et fromage, l'huile, les olives et même les «nourritures obscènes (sperma, menstruum)» (Vogel $1980,87)^{34}$. C'est bien un trait caractéristique du mouvement de Jésus et du christianisme primitif de mettre les repas communautaires au centre de la vie sociale et rituelle, sans que l'on puisse vraiment distinguer ce qui serait un repas simplement convivial et ce qui serait un repas purement cultuel. Une telle distinction n'a d'ailleurs guère de sens puisque, précisément, tous les repas sont chargés d'une valeur cultuelle ${ }^{35}$.

blables d'Emmaüs et du lac de Tibériade (Jn 21,1-14), Jésus se met à leur table. Le Vivant qui n'était plus dans le tombeau prend ainsi congé d'eux dans la convivialité, demeurant présent dans le faire mémoire de la joie de vivre. » (Vouga 2011, 257)

34. «Lait et laitages sont consommés seuls ou en association avec le pain et le miel (ou l'eau). Ainsi chez les artotyrites: Passio Perpetuae et Felicitatis c[hapitre] 4. Le lait mélangé au miel et l'eau font partie, avec le binôme pain et vin, de l'Eucharistie baptismale. Les fragments de Hauler sont explicites à ce sujet. [...] Huile et olives sont consommées seules ou en association avec du pain et du vin eucharistiée, ou encore avec du fromage. Les Fragments de Hauler ne font pas de distinction entre la bénédiction sur le pain et la coupe d'une part, sur l'huile, les olives et le fromage d'autre part. [...] Mentionnons enfin les nourritures obscènes (sperma, menstruum) utilisées dans certains cercles gnostiques, sur lesquelles nous renseignent, entre autres, la Pistis Sophia 147 et Épiphane, Haer, 26.» (Vogel 1980, 86-87)

35. Même si Michel Kunzler prétend lire une distinction entre agape et Repas du Seigneur déjà dans la première lettre de Paul aux Corinthiens. "Ainsi les dénominations bibliques "fraction du pain” et "Repas du Seigneur" soulignent le caractère de repas de la célébration eucharistique. Par "fraction du pain", les passages correspondants de l'Écriture (p. ex. Ac 20,7; 1 Co 10,16ss; 11, 17-34) entendent aussi bien l'agape, 
Ainsi, les Actes des Apôtres désignent tous les repas par une double

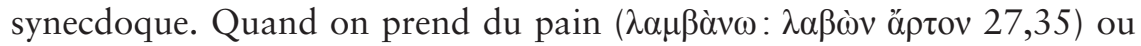

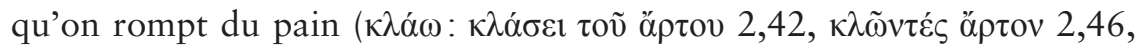

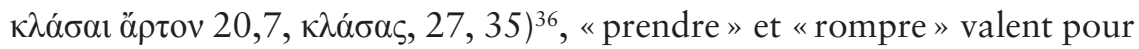
"manger ensemble» et «pain» vaut pour «repas». Et ils insistent sur ces repas quand ils mettent en scène la vie de la première communauté «chrétienne » à Jérusalem où la communion fraternelle, la fraction du pain et la prise de nourriture se font tant au temple qu'à la maison:

${ }^{42}$ [Les nouveaux convertis] étaient assidus à l'enseignement des apôtres et à la communion fraternelle, à la fraction du pain et aux prières. [...] ${ }^{46}$ Unanimes, ils se rendaient chaque jour au temple; ils rompaient le pain à domicile, prenant leur nourriture dans l'allégresse et la simplicité du cœur. (Ac 2,42.46)

Ils montrent comment, un repas communautaire peut devenir l'occasion d'un enseignement, ainsi que le fait Paul à Troas en Asie Mineure: «Le premier jour de la semaine, alors que nous étions réunis pour prendre le pain, Paul, qui devait partir le lendemain, adressait la parole aux frères et il avait prolongé l'entretien jusque vers minuit. » (Ac 20,7).

Enfin, ils présentent Paul qui transforme la nécessité de s'alimenter après un jeune forcé pour cause de tempête en une célébration dont les gestes évoquent les derniers repas de Jésus (prendre du pain — rendre grâce $^{37}$ - rompre - manger), sans que l'on puisse pour autant y lire une réitération de la Cène:

${ }^{33} \mathrm{En}$ attendant le jour, Paul a engagé tout le monde à prendre de la nourriture: "C'est aujourd'hui le quatorzième jour que vous passez dans l'expec-

le repas en commun des premiers chrétiens, que le repas eucharistique. Mais l'appellation "Repas du Seigneur" prétend faire une distinction plus précise entre le repas fraternel, d'allure religieuse, des croyants, et le Repas du Seigneur proprement dit, qui doit être différencié de l'agape, comme l'exprime Paul dans sa première Épître aux Corinthiens. La problématique qui y est abordée (11, 20-22. 33-34) concernant la distinction agape-Repas du Seigneur aboutit à la séparation des deux. Selon 1 Co 11,29 , il s'agit du discernement urgent et vital de la spécificité du Corps eucharistique du Seigneur d'avec toute autre nourriture. (Kunzler 1997, 267). Mais nous avons vu que l'expression utilisée par Paul ( Ce n'est pas le repas du Seigneur que vous prenez») ne désigne pas un repas spécifique, mais la bonne manière de partager la nourriture.

36. Les actes des Apôtres utilisent exactement les verbes que les Évangiles utilisent pour désigner les gestes de Jésus dans les récits de ses derniers repas.

37. Remarquons que cet épisode est le seul à inclure une "eucharistie " explicite et

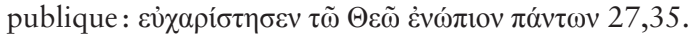


tative sans manger, et vous ne prenez toujours rien. ${ }^{34}$ Je vous engage donc à reprendre de la nourriture, car il y va de votre salut. Encore une fois, aucun d'entre vous ne perdra un cheveu sur sa tête." ${ }^{35}$ Sur ces mots, il a pris du pain, a rendu grâce à Dieu en présence de tous, l'a rompu et s'est mis à manger. ${ }^{36}$ Tous alors, reprenant courage, se sont alimentés à leur tour. (Ac 27,33-36)

Ce que les Actes mettent en récit, la Doctrine des douze apôtres ou Didachè (rédigée "à partir de traditions dont les plus anciennes datent du $1^{\text {er }}$ siècle» (Collin 2006, 54 ${ }^{38}$ ), l'érige en règles. Elle fixe le déroulement de ce qu'elle qualifie d'eucharistie, une autre synecdoque qui désigne le tout d'un repas par le nom de l'une de ses parties: le fait de rendre grâce. Car dans la Didachè, l'eucharistie n'est pas encore le repas symbolique et purement liturgique qu'elle deviendra au cours du deuxième siècle. Le terme indique plutôt le sens que les premiers chrétiens donnent à leurs repas communautaires, ceux qui sont nommés «agapes»(de ảyó $\eta$, amour $\left.{ }^{39}\right)$.

38. Sur le contexte de rédaction de la Didachè, il reste plus de doutes que de certitudes: «Pour tout dire, la Didachè apparaît comme un recueil de traditions différentes, qui ont été rassemblées à une certaine époque par un auteur anonyme qu'il est impossible d'identifier.» (Rordorf 1976, 20)

39. L'expression vient de la lettre de Jude: ${ }^{12}$ Ces gens-là sont les écueils de vos repas

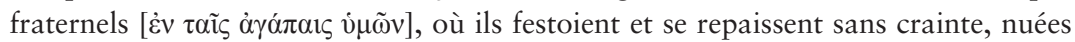
sans eau emportées par les vents, arbres de fin d'automne sans fruits, deux fois morts, déracinés. " (Jude 12). Les chrétiens reprennent du judaïsme et la valeur religieuse du repas et la manière de la signifier: "Chaque fois que le repas comportait un nombre suffisant de convives (parfois trois, parfois dix), on prononçait les prières de bénédiction. Avant le repas, on bénissait le pain et le vin (lorsqu'on en avait); si l'on en croit la tradition sur Schammaï, on bénissait d'abord le vin et ensuite le pain. Puis on mangeait ensemble. Après le repas, on prononçait une longue prière de bénédiction à laquelle les étrangers, les femmes, les esclaves et les enfants ne pouvaient assister. » (Rordorf 1976, 16-17). Mais le repas chrétien tire aussi sa dimension religieuse de la commensalité des repas rituels grecs (Keating, 1969; Smith et Taussig, 1990) et l'eucharistie emprunte aux pratiques romaines: «La forme du repas rituel a posé aux théoriciens un problème d'héritage culturel et cultuel général du plus grand intérêt. On peut le résumer dans cette égalité de scénario: le fidèle des cultes monothéistes païens acclimatés dans la Rome tardive communie, en un banquet de mort et de résurrection du dieu, avec le dieu mourant par deux rites qui sont la fraction du pain et la libation ou aspersion; deux gestes communs à tous sacrifices antiques, la "communion" au dieu mourant aurait été une forme déjà adoptée dans la Rome païenne. [...] Le symbolisme des cérémonies à Mitra est évidemment à prendre en compte d'un autre point de vue. Le pain rompu et l'aspersion sanglante de l'initié offrent en tous cas un modèle rituel concurrent avec le formalisme du repas juif. » (Schefer 2007, 143). Que ce soit pour le récit de la Cène dans les Évangiles ou pour la scénarisation de l'eucharistie dans l'Église primitive, «il faut retenir la probabilité de cet héritage 
J'en trouve un fort indice dans la seconde des deux «prières eucharistiques» que propose la Didachè. La phrase qui l'introduit confirme à la fois l'existence d'une «action de grâce » sur la nourriture et son inscription dans un repas communautaire:

10,1. Après vous être rassasiés, rendez grâce de cette manière: 2 . Nous te rendons grâces, Père saint, Pour ton saint nom Que tu as fait habiter dans nos cœurs, Et pour la connaissance, la foi et l'immortalité Que tu nous as révélées par Jésus ton serviteur, Gloire à toi dans les siècles! (Didachè 10,1-2; Rordorf 1998, 181)

La première prière, quant à elle, mentionne explicitement les aliments utilisés pour cette «eucharistie» et précisent leurs valeurs symboliques. On remarquera qu'il n'est fait aucune référence à la Cène, ni à la trahison, ni à la crucifixion, ni au corps, ni au sang et que l'eucharistie n'est pas fondée, au moins pas explicitement, sur des paroles d'institution attribuées à Jésus.

9 , 1. Pour l'eucharistie, rendez grâce de cette manière: 2. D'abord pour la coupe: Nous te rendons grâce, notre Père, Pour la sainte vigne de David, ton serviteur, Que tu nous as révélée par Jésus, ton serviteur, Gloire à toi dans les siècles! 3. Puis pour le pain rompu: Nous te rendons grâce, notre Père, Pour la vie et la connaissance Que tu nous as révélées par Jésus, ton serviteur, Gloire à toi, dans les siècles! 4. Comme ce pain rompu, disséminé sur les montagnes, a été rassemblé pour être un, que ton Église soit rassemblée de la même manière des extrémités de la terre dans ton royaume. Car c'est à toi qu'appartiennent la gloire et la puissance par Jésus-Christ dans les siècles! (Didachè 9,1-4; Rordorf 1998, 177-179)

C'est chez Ignace d'Antioche, au tout début du $\mathrm{II}^{\mathrm{e}}$ siècle, que le terme «eucharistie» prend «un sens technique et signifie la commémoration liturgique de la Cène au cours de laquelle les oblats sont convertis au corps et au sang du Christ pour être pris en nourriture au moment de la communion. ( (Johanny 1976, 57-58). Mais il faut encore attendre cinquante ans pour avoir le premier témoignage écrit qu'elle forme une célébration totalement autonome, détachée du repas communautaire. Et c'est Justin qui, dans sa Première Apologie (écrite autour de 150), en fait les premières mentions écrites. Au chapitre 65, il raconte l'eucharistie baptismale et au chapitre 67, il décrit l'eucharistie dominicale:

formel de rituels païens qui ont joué le rôle précis de “déjudaïser" pour le premier christianisme les rites chrétiens. » (Schefer 2007, 143) 
[3] Le jour qu'on appelle le jour du soleil, tous, dans les villes et à la campagne, se réunissent dans un même lieu: on lit les mémoires des apôtres et les écrits des prophètes, autant que le temps le permet. [4] Quand le lecteur a fini, celui qui préside fait un discours pour avertir et pour exhorter à l'imitation de ces beaux enseignements. [5] Ensuite nous nous levons tous et nous prions ensemble à haute voix. Puis, comme nous l'avons déjà dit, lorsque la prière est terminée, on apporte du pain avec du vin et de l'eau. Celui qui préside fait monter au ciel les prières et les eucharisties [au sens ici d'actions de grâces] autant qu'il peut, et tout le peuple répond par l'acclamation Amen. Puis a lieu la distribution et le partage des choses consacrées à chacun et l'on envoie leur part aux absents par le ministère des diacres. (Première Apologie LXVII,3-5 ; Justin et Pautigny 1904, 153)

Entre les deux, au chapitre 66, il indique le sens de ce repas. Et c'est alors qu'il établit un lien explicite avec la Cène de Jésus:

[3] Les apôtres, dans leurs Mémoires, qu'on appelle Évangiles, nous rapportent que Jésus leur fit ces recommandations: il prit du pain, et ayant rendu grâces, il leur dit: "Faites ceci en mémoire de moi: ceci est mon corps. » Il prit de même le calice, et ayant rendu grâces, il leur dit: "Ceci est mon sang.» Et il les leur donna à eux seuls. (Première Apologie LXVI,3; Justin et Pautigny 1904, 151) ${ }^{40}$

Et c'est ainsi que la Cène est devenue, pour Jésus, la dernière et, pour les chrétiens, la première.

\section{Références}

Aus, R. D. (2000), "The Road to Emmaus (Luke 24: 13-35)», dans The Stilling of the Storms. Studies in Early Palestinian Judaic Traditions ISFCJ, (International Studies in Formative Christianity and Judaism), Binghamton, Global Publication.

Bailly, A. (1901), Abrégé du dictionnaire grec-français, Paris, Hachette.

Carrez, M., et F. Morel $\left(1980^{2}\right)$, Dictionnaire grec-français du Nouveau Testament, Neuchâtel/Paris, Delachaux et Niestlé/Cerf.

40. Justin prend soin de "démitraïser" cette eucharistie: "[4] Les mauvais démons ont imité cette institution dans les mystères de Mithra: on présente du pain et une coupe d'eau dans les cérémonies de l'initiation et on prononce certaines formules que vous savez ou que vous pouvez savoir." Première Apologie LVI,4 (Justin et Pautigny, 1904, p. 151). 
Bauer, W., W. F. Arndt et F. W. Gingrich (1957), A Greek-English Lexicon of the New Testament and Other Early Christian Literature. A Translation and Adaptation of Walter Bauer's Griechisch-Deutsches Wörterbuch zu den Schriften des Neuen Testaments und der übrigen urchristlichen Literatur, Fourth Revised and Augmented Edition, 1952, Chicago, University of Chicago Press.

Caseau, B. (2002), "L'Eucharistie au centre de la vie religieuse des communautés chrétiennes (fin du $\mathrm{IV}^{\mathrm{e}}$ au $\mathrm{X}^{\mathrm{e}}$ siècle) », dans M. BrouARD, dir., Eucharistia. Encyclopédie de l'Eucharistie, Paris, Cerf, p. 105-144.

Collin, M. (2006), «La Bible dans la liturgie chrétienne des premiers siècles ", dans M. Klöckener, B. Bürki et A. Join-Lambert, dir., Présence et rôle de la Bible dans la liturgie, Fribourg, Academic Press Fribourg, p. 4368.

Gaffiot, F. (1934), Dictionnaire Latin-Français, Paris, Hachette.

Genest, O. (2005), «De la Cène de Jésus à l'Eucharistie des chrétiens », dans J.-G. NAdeau. dir., La liturgie, mise en scène ou entracte?, Montréal, Novalis, p. 35-50.

Geoltrain, P., J.-D. Kaestli, J.-M. Roessli et S. J. Voicu (2005), Écrits apocryphes chrétiens II, Paris, Gallimard (La Pléiade).

Humphreys, C. J. (2011), The Mystery of the Last Supper: Reconstructing the Final Days of Jesus, Cambridge /New York, Cambridge University Press, consulté à : <http.//assets.cambridge.org/97805215/17553/ cover/9780521517553.jpg>.

Johanny, R. (1976), "Ignace d'Antioche", dans W. Rordorf, dir., L'Eucharistie des premiers chrétiens, Paris, Beauchesne, p. 53-74.

Justin et Pautigny, L. (1904), Apologies, Texte grec, traduction française, introduction et index par Louis Pautigny, Paris, A. Picard et fils, consulté à : <http://gallica.bnf.fr/ark: /12148/bpt6k1121309>.

Keating, J. F. (1969), The Agape and the Eucharist in the Early Church. Studies in the History of the Christian Love-Feasts, New York, AMS Press.

Kunzler, M. (1997) [allemand 1995], La liturgie de l'Église / trad. par J. Voss, Luxembourg, Cerf (Amateca).

Mainville, O. (2007), Les christophanies du Nouveau Testament. Historicité et théologie, Montréal, Médiaspaul (Sciences bibliques 19).

McGowan, A. B. (1999), Ascetic Eucharists. Food and Drink in Early Christian Ritual Meals. Oxford/New York, Clarendon Press/Oxford University Press, consulté à: <http.//www.loc.gov/catdir/enhancements/ fy0605/98042962-d.html>. 
Nestle, E. et B. Aland (2012), Novum testamentum Graece, Stuttgart, Dt. Bibel-Ges.

Rordorf, W. (1976), et al., L'Eucharistie des premiers chrétiens, Paris, Beauchesne.

Rordorf, W. et A. Tuilier $\left(1998^{2}\right)$, La Doctrine des Douze Apôtres (Didachè), Paris, Cerf.

Rosenblum, J. (2010), Food and Identity in Early Rabbinic Judaism, Cambridge/New York, Cambridge University Press.

SCHEFER, J.-L. (2007), L’hostie profanée histoire d'une fiction théologique, Paris, Pol.

Segond, L. et Alliance biblique universelle (2002), La Nouvelle Bible Segond, édition d'étude, Paris, Alliance biblique universelle.

Smith, D. E. et H. Taussig (1990), Many Tables, the Eucharist in the New Testament and Liturgy Today, London/Philadelphia, SCM Press/Trinity Press International.

Vloberg, M. (1946), L'Eucharistie dans l'art, Grenoble Paris, B. Arthaud.

Vogel, C. (1980), "Symboles cultuels chrétiens, Aliments et boissons", Concilium, 152, p. 83-89.

Vouga, F. (2011), «L'absence du corps vivant, l'absence du corps mort et la présence réelle du ressuscité. La gastronomie de Pâques dans l'Évangile de Luc ", dans A. Gignac, A. Gagné et S. Paquette Lessard, dir., Le Vivant qui fait vivre. Esprit, éthique et résurrection dans le Nouveau Testament. Mélanges offerts à la professeure Odette Mainville, Montréal, Médiaspaul (Sciences bibliques 22), p. 239-259.

\section{Résumé}

Il est d'usage de qualifier le repas que Jésus prend avec ses disciples la veille de son arrestation de «dernier repas». Or, il n'en est rien. Une lecture attentive des évangiles de Luc et de Jean montre que Jésus mange encore après sa mort: au moins du poisson, peut-être du pain et du miel. Un tel constat pourrait relever de l'anecdote et le travail qui y conduit d'un pur souci d'érudition. Sauf qu'ils ont des conséquences pratiques et des impacts contemporains. Car l'insistance sur une «dernière cène» où Jésus partage le pain et le vin a conduit l'Église chrétienne à survaloriser l'eucharistie comme seul repas liturgique, alors que le christianisme primitif semble avoir fait de tous les repas l'occasion de rencontrer le Ressuscité. 


\section{Abstract}

It is common to call "the Last Supper» the meal Jesus had with his disciples just before his arrest. But careful readings of the gospels of Luke and John show that Jesus keeps eating after his death: some fish, at least - maybe even bread and honey. This could be considered as an anecdote and a research on the subject considered as a purely intellectual concern. Except that this has practical consequences - and still does. As a matter of fact, insisting on that "Last Supper», when Jesus shares wine and bread, has brought the Church to overvalue the Eucharist as the only liturgical meal, even after primitive Christianity appears to have found in every meal an occasion to meet the Resurrected. 